\title{
LEXICAL INFLUENCES ON THE MCGURK EFFECT
}

\author{
Lawrence Brancazio
}

Department of Psychology, Northeastern University, Boston, MA 02115

\begin{abstract}
The purpose of this research was to explore the interrelationship of audiovisual speech perception and spoken word recognition. I tested whether an index of audiovisual integration, the "McGurk effect," would be influenced by the lexical status of the stimuli. There was a significant increase in the McGurk effect when the visually-influenced percept formed a word than when it formed a nonword, and an increase in the effect when the auditory stimulus was a nonword compared to when it was a word. A second experiment ruled out a response-bias account of these findings by demonstrating a similar effect in nonword stimuli that differed in their number of word "neighbors." I discuss the implications of these findings for theories of audiovisual speech perception.
\end{abstract}

\section{INTRODUCTION}

Most research examining audiovisual integration of speech has only involved perception of nonsense syllables. Few studies have investigated the potential interactions between the processes of audiovisual integration and lexical knowledge. One possible approach is to investigate word recognition from degraded auditory signals, visual speech signals, and the combination of the two signals in order to gauge the enhancement of recognition due to audiovisual integration [1]. A complementary approach is to compare phonetic categorization of audiovisually presented speech in word and nonword contexts. In the auditory domain, Ganong [2] found that stimuli in the mid-range of an acoustic continuum varying from $/ \mathrm{gI} /$ to $/ \mathrm{kI} /$ were more likely to be identified as containing /g/ if a final $/ \mathrm{ft} / \mathrm{t}$ was appended (thus creating a "gift"-"kift" continuum) than if a final /s/ was appended (thus creating a "giss"-"kiss" continuum). This finding is taken as evidence for interactive models of speech perception [3], although some autonomous models are able to account for the finding as well $[4,5]$.

The present research addresses the question of whether a phenomenon analogous to the "Ganong effect" will occur for audiovisually presented speech. In order to create perceptually ambiguous stimuli, I used audiovisually discrepant speech--that is, stimuli that produce the "McGurk effect" [6]. There is evidence that percepts arising from audiovisually discrepant stimuli have a degree of perceptual ambiguity, evidenced by performance in discrimination tasks [7], by relatively central ratings on continuous scales with different phonemes as endpoints [8], and by the fact that the McGurk effect usually occurs on only some percentage of trials.

I examined the incidence of the McGurk effect in stimuli (audiovisually discrepant only in the initial consonant) in which the auditory portion of the stimulus and the integrated percept each formed either a word or a nonword. Both factors might influence the McGurk effect; auditory wordness might weaken the effect, whereas integrated-percept wordness might strengthen the effect. A similar study in Finnish by Sams et al. [9] failed to find a lexical influence on the McGurk effect. However, a positive finding would indicate that audiovisual speech perception, like auditory perception, is constrained by higher-order knowledge, and further that audiovisual discrepancy produces perceptual ambiguity that is not fundamentally different from the ambiguity produced by manipulation of acoustic cues. Moreover, it would counter the notion [10] that speech perception from audiovisual signals, particularly incongruent ones, represents a "special case" that may not generalize from nonsense syllables to more meaningful conditions.

\section{EXPERIMENT 1}

The purpose of Experiment 1 was to establish whether, for audiovisually discrepant stimuli, the lexical status of the auditory stimulus and of the integrated percept (which was the same as the lexical status of the original production of the visually presented syllable) affect the incidence of the McGurk effect. Goodness ratings (of how well the percept matched the selected phonetic category) were also collected to assess the degree of perceptual ambiguity. 


\subsection{Methods}

\section{Subjects}

Sixty undergraduates at the University of Connecticut participated in the experiment for course credit. All were native speakers of English and reported normal hearing and normal or corrected vision.

\section{Materials}

Sixty-four pairs of syllables, in which the rimes of the members of a given pair were identical, were selected. The initial-consonant contrasts used were $/ \mathrm{b} /-/ \mathrm{d} /, / \mathrm{m} /-/ \mathrm{n} /, / \mathrm{b} /-/ \mathrm{v} /$, and $/ \mathrm{p} /-/ \mathrm{t} /$ (16 of each). Equal numbers of pairs were selected in which both syllables formed words; neither formed a word; the bilabial pair member $(/ \mathrm{b} /, / \mathrm{m} /$, or $/ \mathrm{p} /)$ formed a word while the other $(/ \mathrm{d} /, / \mathrm{n} /, / \mathrm{t} /$, or $/ \mathrm{v} /)$ did not; and the bilabial pair member did not form a word but the other did. A male speaker's productions of each of the 128 syllables were videotaped and stimuli were digitized; all four of the possible audiovisual configurations (two audiovisually congruent, two discrepant) were created. Examples are in Table 1.

\begin{tabular}{|l|l|l|l|}
\hline Audio & Video & Audio Lex. & Video Lex. \\
\hline \hline BELT & DEALT & W & W \\
\hline BESK & DESK & NW & W \\
\hline BEG & DEG & W & NW \\
\hline BEDGE & DEDGE & NW & NW \\
\hline
\end{tabular}

Table 1. Examples of Experiment 1 stimuli.

\section{Procedure}

Stimuli were presented by computer; participants entered responses by keyboard. They first typed in the letter or letters corresponding to the initial consonant(s) they heard at the beginning of each syllable and then gave a goodness rating for that judgment, using a scale from 1 (poor) to 5 (excellent). Each participant was only shown one of the four audiovisual configurations of each syllable pair (64 stimuli total). Each subject received equal numbers of congruent and discrepant trials.

\subsection{Results}

Accuracy on audiovisually congruent trials was high (98.7\% correct) and will not be reported further. For audiovisually discrepant trials, the primary dependent measure was the percentage of "visually-dominant" responses (responses with the visually-specified place of articulation and voicing and manner consistent with the acoustic signal). "Visually-dominant" responses were given on $49.7 \%$ of trials, and "Auditorily-determined" responses (matching the auditory portion of the stimulus) were given on $30.8 \%$ of trials. A 2 ("Audio Lexicality": whether the auditory stimulus formed a word or not) X 2 ("Video Lexicality": whether the visually-dominant percept formed a word) X 2 ("Place of Articulation" or POA: whether the auditory stimulus was bilabial or not; in the latter case the visual stimulus was bilabial) ANOVA on visually-based responses was conducted by subjects and items. The pattern of results is shown in Figure 1. The McGurk effect was strongest when the visually-dominant percept formed a word but the auditory percept formed a nonword, weakest in the opposite case, and intermediate for the cases in which both formed words or both formed nonwords. There was a significant $10.9 \%$ effect of Audio Lexicality $(\mathrm{F} 1[1,59]=55.68, \mathrm{p}<.0001 ; \mathrm{F} 2[1,120]=5.6, \mathrm{p}<$ $.05)$ and a significant $21.8 \%$ effect of Video Lexicality $(\mathrm{F} 1[1,59]=117.86, \mathrm{p}<.0001 ; \mathrm{F} 2[1,120]$ $=20.65, \mathrm{p}<.0001)$. The two lexical factors did not interact. There was also a main effect of POA (F1[1, $59]=25.44, \mathrm{p}<.0001 ; \mathrm{F} 2[1,120]=12.09, \mathrm{p}<.001)$ with a stronger McGurk effect when the audio was a bilabial than when the video was bilabial. A complementary pattern of results emerged for the auditory-based responses with more auditory-based responses when they formed a word than when they formed a nonword and fewer when the visuallyinfluenced percept formed a word than a nonword.

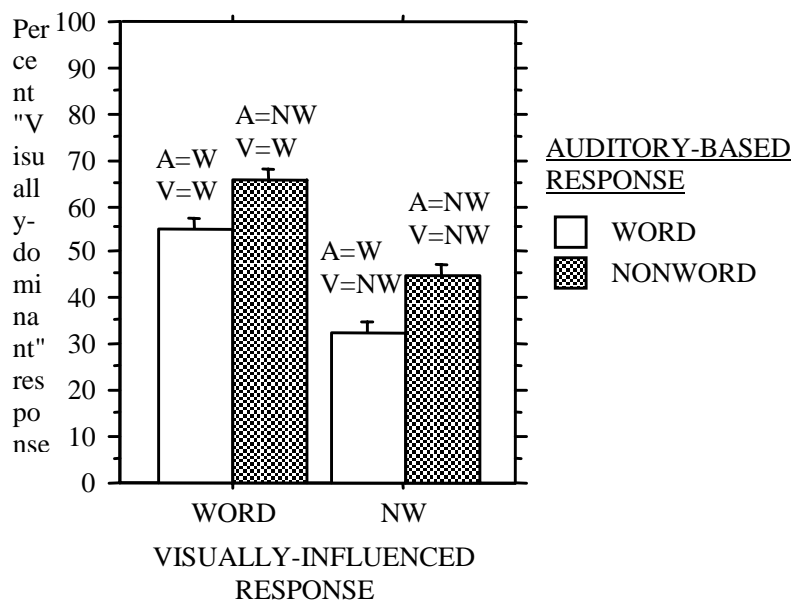

Figure 1. Lexical influences on the percentage of "visuallydominant" responses in Experiment 1. 
To examine the degree to which audiovisual discrepancy produced perceptual ambiguity, the goodness ratings were analyzed. The ratings for three categories of identification responses were compared: correct responses on audiovisually congruent trials; visually-influenced responses ("McGurk" responses) on discrepant trials; and auditorily-based responses on discrepant trials. The means for each condition are presented in Table 2. There were significant differences among the conditions $(\mathrm{F}[2,118]=146.38$, $\mathrm{p}<.0001)$, and each mean differed significantly from the others (smallest $F[1,59]=31.02, p<.0001$ ). Ratings were significantly higher for congruent than for incongruent stimuli (regardless of classification). Ratings were also higher for "McGurk" responses than for "non-McGurk" responses on the discrepant trials.

\begin{tabular}{|l|c|c|}
\hline Response & $\begin{array}{c}\text { Mean Goodness } \\
\text { Rating }\end{array}$ & $\begin{array}{c}\text { Standard } \\
\text { Deviation }\end{array}$ \\
\hline \hline Congruent - Correct & 4.25 & .483 \\
\hline $\begin{array}{l}\text { Discrepant - Visually- } \\
\text { dominant ("McGurk") }\end{array}$ & 3.28 & .574 \\
\hline $\begin{array}{l}\text { Discrepant - Auditorily- } \\
\text { based ("non-McGurk") }\end{array}$ & 2.81 & .805 \\
\hline
\end{tabular}

Table 2. Mean goodness ratings in Experiment $1(1=$ poor; $5=$ excellent)

\subsection{Discussion}

Experiment 1 demonstrated a robust lexical influence on the McGurk effect in the predicted direction. The effect was quite strong; there was a $33.1 \%$ difference in the percentages of McGurk responses between the strongest case (audio=nonword, video $=$ word) and the weakest (audio=word, video=nonword). Importantly, the lexicality of the auditory signal and of the visually-dominant percept (that is, the integrated percept incorporating the visually-specified place of articulation) both influenced the McGurk effect, indicating that the lexical effect was not specifically auditory; in fact, the lexical status of the visuallydominant percept had a numerically greater main effect. The goodness ratings produced interesting results as well. The expected finding of lower ratings for discrepant than for congruent tokens lends credence to the view that audiovisual discrepancy produces a degree of perceptual ambiguity (which, in turn, provides an appropriate environment for lexical effects to emerge). Additionally, the finding of lower ratings for non-McGurk responses than for McGurk responses on the discrepant trials is interesting because it indicates that these auditory responses do not necessarily result from subjects discounting (whether consciously or not) the visually presented stimulus. Instead, it suggests that the visual token influences perception, even if the influence is not reflected in phonetic categorization.

These stimuli have also been presented in an experiment in which subjects made speeded, forcedchoice responses, and the basic pattern of results replicated (although the overall lexical effects were somewhat smaller in magnitude). Reaction times in the follow-up experiment corresponded to the goodness ratings, such that responses were faster to congruent than discrepant stimuli, and McGurk responses were faster than non-McGurk responses on the discrepant trials. Additionally, lexical effects were greater for slow than for fast responses, replicating a typical outcome in the auditory Ganong paradigm [11].

This finding of a significant lexical influence on the McGurk effect is at odds with the results of Sams et al. [9]. The disparity between the outcomes might stem from the fact that individual stimuli differ substantially in their susceptibility to the McGurk effect for reasons unrelated to lexical status (for example, depending on vowel context), and there may have been systematic differences in both experiments between stimuli in which the audio formed a word and in which it formed a nonword. Possibly, such differences obscured any lexical effects in Sams et al.'s study, which utilized fewer stimuli than the present experiment. This possibility aside, it should be noted that some studies have failed to produce evidence for a lexical effect in the auditory domain as well, and the reasons for this cross-experiment variability are not well understood [12]. Such variability is therefore not unexpected in the audiovisual domain.

\section{EXPERIMENT 2}

Although the findings of Experiment 1 are striking, they should be regarded with some caution. First, it is possible that the lexical effects are due to a postperceptual response bias rather than a perceptual effect (which could still be a "bias" in signal-detection terms). That is, the participants may have simply been exhibiting a preference to give word-forming responses; this would render the result rather uninteresting. Second, different stimuli were used in the four lexical cells (i.e., audio=word, video=word; audio=nonword, video=word; etc.), and it is possible that accidental, uncontrolled stimulus differences 
contributed to the apparent lexical effects (although a large number of different stimuli were used in each cell, and the effects were significant in items analyses). Both of these issues were addressed in Experiment 2.

In order to test the response-bias interpretation, I adopted an approach of Newman et al. [13] for Experiment 2. Newman et al. demonstrated a lexical neighborhood effect in nonwords akin to the Ganong effect for auditory continua by demonstrating a shift in the voiced-voiceless boundary between a "beysh""peysh" continuum and a "beyth"-"peyth" continuum. "Beyth" has more word neighbors (and a greater sum of the Kucera-Francis frequencies of its neighbors) than "peysh," whereas "peyth" has more neighbors and a greater neighborhood frequency than "beyth," where a neighbor is defined as any word that can be created by adding, deleting, or changing one phoneme in any position in the syllable. This finding demonstrates lexical involvement in phonetic categorization without explicit recognition of words and thus is unlikely to be due to a post-perceptual response bias favoring words.

Accordingly, Experiment 2 utilized stimulus sets with minimal pairs differing in place of articulation of the initial consonant (instead of voicing contrasts as used by Newman et al.). Thus, the McGurk effect in a stimulus such as audio "besh" + video "desh" ("desh" has a larger neighborhood frequency than "besh") was compared to the effect in a stimulus such as audio "betch" + video "detch" ("betch" has a higher neighborhood frequency than "detch"). Lexical sets (where either the audio or the video, but not both, formed a word) were also used for comparison and for replication of Experiment 1.

Experiment 2 controlled for stimulus differences by using the same acoustic CV portions in the contrasting stimuli (i.e., the same "be" fragment in "besh" and "betch" tokens, using a cross-splicing technique) and the same visual CV portion (one truncated video "de" token for both pairings). Therefore, any lexical or neighborhood effects in this experiment are not due to low-level acoustic or visual differences between stimuli. Therefore, this experiment can better isolate lexical effects than either Experiment 1 or Sams et al.'s [9] study.

\subsection{Methods}

\section{Subjects}

Twenty-five members of the University of Connecticut community participated in the experiment and were paid \$10. All were native speakers of English and reported normal hearing and normal or corrected vision.

\section{Materials}

Three sets of nonwords were created: "besh" - "desh" - "betch" - "detch," "maunth" - "naunth" - "maunt" "naunt," and "powth" - "towth" - "powce" - "towce." The first pair member had a higher neighborhood count and neighborhood frequency than the second member, while the fourth had greater neighborhood statistics than the third. Three lexical sets were also created: "bense" - "dense" - "bench" - "dench," "meck" - "neck" - "mess" - "ness," and "pake" "take" - "pace" - "tace." A male speaker's productions of each token were videotaped, and several acoustic recordings (from a separate session) were made. One token of each video was selected and truncated at the point of maximal vowel opening, so that the videos could be paired with acoustic syllables with different final consonants. Six auditory tokens of each syllable were digitized and the final consonant was replaced by two new final consonants (for example, the $/ \mathrm{k} /$ and /s/ from "meck" and "mess" syllables were respectively removed; new $/ \mathrm{k} / \mathrm{s}$ and $/ \mathrm{s} / \mathrm{s}$ were appended to both syllables to create two new "meck" tokens and two new "mess" tokens). All tokens were then dubbed with all of the videos in that set: thus, the spliced auditory "meck," "mess," "neck," and "ness" tokens were paired with video "me" tokens truncated from "meck" and "mess," and with video "ne" tokens truncated from "neck" and "ness."

\section{Procedure}

Stimuli were presented by computer; participants entered their responses by clicking with the mouse in boxes on the monitor corresponding to different responses. The response options were "B," "D," "M," "N," "P," "T," "BD," "MN," "PT," "TH," "K," and "L."

\subsection{Results}

As in Experiment 1, accuracy on audiovisually congruent trials was high (96.5\% correct) and will not be reported further. For audiovisually discrepant trials, the dependent measure of interest was the percentage of "visually-dominant" responses (combining the auditorily-specified manner and voicing and the visually-specified place of articulation on the initial consonant, and the auditorily-specified vowel and final consonant). Overall, visually-dominant responses were given on 
$30.6 \%$ of discrepant trials, and auditory responses were given on $40.3 \%$ of trials. Fusion ("TH" for the /b/-/d/ and /p/-/t/ and "L" for the $/ \mathrm{m} /-/ \mathrm{n} /$ pairings) or combination ("BD," "MN," and "PT") responses were given on $25.8 \%$ of the trials. Thus, the McGurk effect was weaker than in the previous experiment. Pilot testing revealed that this was partly due to the truncation procedure; the same stimuli without video truncation produced a much stronger effect. The percentage of visually-dominant responses was analyzed in a 2(Lexical Set: Word-Nonword vs. Neighborhood) X 2 (Asymmetry: Audio+, for Audio word/high neighborhood - Video nonword/low neighborhood stimuli vs. Video+, for Audio nonword/low neighborhood - Video word/high neighborhood stimuli) X 3 (Consonant Pair: /b/-/d/ vs. $/ \mathrm{m} /-\mathrm{n} / \mathrm{vs} . / \mathrm{p} /-/ \mathrm{t} /$ ) X 2 (POA: bilabial audio with an alveolar video, vs. alveolar audio with a bilabial video) ANOVA by subjects and items. The crucial aspects of the analysis are the main effect of Asymmetry and its interaction with Lexical Set (to test whether there are comparable effects for the Lexical and Neighborhood sets).

The results are presented in Figure 2. There was a main effect of Asymmetry $(\mathrm{F} 1[1,24]=12.21$, p < $.005 ; \mathrm{F} 2[1,60]=28.44, \mathrm{p}<.0001)$ with more visually-dominant responses when the auditory token was a nonword or a low-neighborhood nonword (Video+) than when it was a word or a highneighborhood nonword (Audio+). Importantly, Asymmetry did not interact with Lexical Set $(\mathrm{F} 1<1$, F2 < 1), meaning that both the Lexical and the Neighborhood effects were significant. As the figure demonstrates, however, both effects were quite small (4.7\% lexical effect; $3.3 \%$ neighborhood effect). Moreover, the effects did not carry over to the percentage of auditory-based responses, in which there was an interaction between Asymmetry and Lexical Set $(\mathrm{F} 1[1,24]=11.73, \mathrm{p}<.005 ; \mathrm{F} 2[1,60]=$ $42.78, \mathrm{p}<.0001$ ), with a lexical effect but no neighborhood effect. However, analyses of the fusion and combination responses did reveal neighborhood effects.
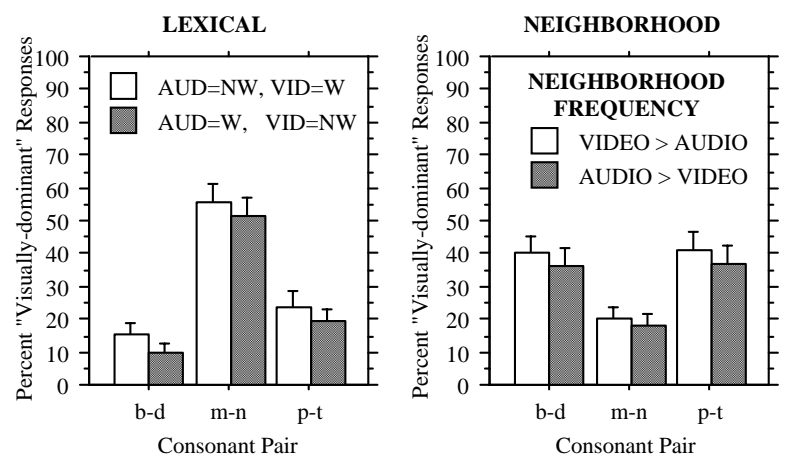

$\mathrm{Fi}$

gure 2. Lexical and Neighborhood effects on percentage of "visually-dominant" responses in Experiment 2.

\subsection{Discussion}

The results of Experiment 2 demonstrate two important points. One is that the lexical influence on the McGurk effect observed in Experiment 1 occurs even when stimuli are perfectly matched on physical properties. The other is that the lexical influence does not depend on conscious recognition of words. A plausible interpretation of the results is that nonwords in the Neighborhood manipulation partially activated word representations, and that phonetic categorization of the discrepant stimuli was influenced by these partial activations; the more words that were partially activated (and the higher the frequency of these words), the greater the top-down influence on categorization, and hence on the McGurk effect. This neighborhood effect strongly suggests that the lexical influence on the McGurk effect was not strictly due to a post-perceptual response bias favoring words; at least some component of the lexical effect was perceptual.

The lexical effect in Experiment 2 was notably smaller than that of Experiment 1. This may be due to the fact that the same syllables were repeated many times in Experiment 2, whereas each syllable was presented only once in Experiment 1. This repetition may have weakened the lexical influence by increasing the familiarity of the nonwords.

\section{CONCLUSIONS}

This research demonstrates that lexical knowledge plays a significant role in audiovisual speech perception, particularly under conditions in which the auditory and visual signals conflict. How might the findings be explained? One general conclusion is that audiovisual discrepancy gives rise to two (or more) viable phonetic candidates for categorization - that is, it creates phonetic ambiguity - and that top-down 
lexical knowledge has an influence on the resolution of this ambiguity. This conclusion invokes the specific assumption that the process of lexical access operates on an "output" from the process of audiovisual integration. (This does not mean that lexical access cannot begin until integration is resolved; to the contrary, given that the output of the integration process can evolve over the time-course of processing, lexical access could initially operate upon a partial output of integration.) However, it is neutral with respect to other aspects of perceptual architecture concerning the relationship of audiovisual integration and lexical knowledge, as will be discussed below.

In contrast to the preceding conclusion, an alternative account might be that lexical access utilizes the output of pre-integration, modality-specific processing of the auditory and visual signals before or contemporaneous with integration. However, a problem for this account is that lexical access is less efficient in speechreading than in listening, due to larger phonemic equivalence classes in speechreading [1]. Therefore, this latter view would predict that lexicality of the auditory stimulus would matter more than that of the visual stimulus. This prediction was not borne out in Experiment 1; to the contrary, the Video Lexicality factor had a numerically greater impact than Audio Lexicality. Rather, lexical influences appear to involve multiply activated lexical candidates arising via multiply activated phoneme candidates from (incompletely resolved) audiovisual integration.

What about more specific accounts of interactions of lexical knowledge and audiovisual integration? There is a debate whether lexical activation affects low-level phonetic processing [3] or later stages [4, 5] in auditory speech perception. Interactive models and autonomous models can be extended to the audiovisual domain, but the present results do not offer a basis for distinguishing them. It is possible that the lexical effects observed here occur because top-down lexical knowledge affects low-level auditory and visual processing, as well as the integration stage, through interactive connections between levels (following McClelland and Elman [3]). Or, low-level auditory and visual processing could be independent of lexical influences, but the latter influences could come into play at a prototypeevaluation stage, which could also be the point of audiovisual integration as well (following Massaro's FLMP $[4,14]$ ). Third, integration could be unaffected by lexical knowledge, and lexical influences only emerge at a post-integration stage (following
McQueen et al. [5]). This latter account is also concordant with Burnham's [15] Phonetic Plus PostCategorical (3PC) model, with its distinction between integration and categorization.

Various accounts of audiovisual integration will differ in their compatibility with these different accounts. Notably, the direct-realist account [16] and modularist accounts such as the Motor Theory [17] would not predict lexical effects on integration itself, and therefore are more in line with the third account. Further research into the locus of these lexical effects thus may be useful for distinguishing theories of audiovisual speech perception.

\section{REFERENCES}

1. Iverson, P., Bernstein, L.E., and Auer, E.T. "Modeling the interaction of phonemic intelligibility and lexical structure in audiovisual word recognition," Speech Comm (26): 4563, 1998.

2. Ganong, W.F. "Phonetic categorization in auditory word perception," J. Exp. Psych.: Hum. Perc. Perf. (6): 110125,1980

3. McClelland, J.L., and Elman, J.L. "The TRACE model of speech perception,” Cogn. Psych. (18): 1-86, 1986.

4. Massaro, D.W., and Oden, G.C. "Independence of lexical context and phonological information in speech perception," J. Exp. Psych.: Learn. Mem. Cogn. (21): 1053-1064, 1995.

5. McQueen, J.M., Norris, D., and Cutler, A. "Lexical influence in phonetic decision-making: Evidence from subcategorical mismatches," J. Exp. Psych.: Hum. Perc. Perf, in press.

6. McGurk, H., and MacDonald, J. "Hearing lips and seeing voices," Nature (264): 746-748, 1976.

7. Rosenblum, L.D., and Saldan $\neq$ a, H.M. "Discrimination tests of visually influenced syllables," Perc. Psychophys (52): 461-473, 1992.

8. Massaro, D.W., and Ferguson, E.L. "Cognitive style and perception: The relationship between category width and speech perception, categorization, and discrimination, “ Amer. J. Psych. (106): 25-49, 1993.

9. Sams, M., Manninen, P., Surakka, V., Helin, P., and Kättö, R. "McGurk effect in Finnish syllables, isolated words, and words in sentences: Effects of word meaning and sentence context," Speech Comm (26): 75-87, 1998.

10. Easton, R.D. and Basala, M. "Perceptual dominance during lipreading," Perc. Psychophys. (32): 562-570, 1982. 
11. Fox, R.A. "Effect of lexical status on phonetic categorization," J. Exp. Psych.: Hum. Perc. Perf. (10): 526-540, 1984.

12. Pitt, M.A., and Samuel, A.G. "An empirical and metaanalytic evaluation of the phoneme identification task," $J$. Exp. Psych.: Hum. Perc. Perf. (19): 699-725, 1993.

13. Newman, R.S., Sawusch, J.R., and Luce, P.A. "Lexical neighborhood effects in phonetic processing," J. Exp. Psych.: Hum. Perc. Perf. (23): 873-889, 1997.

14. Massaro, D.W. Perceiving Talking Faces: From Speech Perception to a Behavioral Principle, MIT Press, Cambridge, MA, 1998.

15. Burnham, D. "Language specificity in the development of auditory-visual speech perception," n R. Campbell, B. Dodd, and D. Burnham (eds. ), Hearing by Eye II, Psychology Press Ltd., Hove, UK, 27-60, 1998.

16. Fowler, C.A. "An event approach to the study of speech perception from a direct-realist perspective," J. Phon. (14): 3-28, 1986.

17. Liberman, A.M., and Mattingly, I.G. "The motor theory of speech perception revised," Cogn. (21): 1-36, 1985.

\section{ACKNOWLEDGMENTS}

This research was supported by NIH grant HD-01994 to Haskins Laboratories and by a dissertation fellowship from the University of Connecticut. Thanks to Carol Fowler for her guidance throughout the project. 